\title{
Complex THz Reflectance Spectra of Hexogen Measured for Various Surfaces
}

\author{
N. PALKA* \\ Institute of Optoelectronics, Military University of Technology \\ S. Kaliskiego 2, 00-908 Warsaw, Poland
}

\begin{abstract}
In this paper, we report on measurements of reflection spectra of hexogen (RDX) by means of time domain spectroscopy. We analyzed both phase and amplitude of reflected impulses for three cases: detuning, samples with rough surfaces and samples with curved surfaces. It can be concluded that both spectral characteristics can be applied for identification of explosives in the reflection configuration.
\end{abstract}

PACS: 07.57.Ty, 78.20.Ci, 78.40.Pg, 78.40.-q, 78.47.jg

\section{Introduction}

Stand-off detection of many substances, including explosive materials, has been focusing attention for over a few decades. Terahertz technology, due to many unusual features, is a good candidate for this purpose.

Due to high absorption of explosives in $\mathrm{THz}$ range, only reflection geometry seems to be applicable for remote detection. Unfortunately, there are many factors that influence this kind of measurement: surface morphology, size of particles inside a sample, coverings, and geometry of a system. Due to unique properties of time domain spectroscopy (TDS) technique, spectral features of a target can be measured roughly in the range 0.3 3.0 THz. As a result, complex reflectance characteristic of the material, which includes amplitude and phase part, can be determined. A commonly used explosive material - hexogen (RDX) is investigated in this paper due to a fact that it has a very pronounced absorption peak at the frequency $0.82-0.84 \mathrm{THz}$. In this frequency region most TDS systems have the highest sensitivity and performance.

A pure RDX sample pressed to a pellet and a RDX-based plastic explosive were prepared to study influence of the sample position and surface morphology on the reflectance and the reflectance phase.

For measurements we applied a commercially available TDS setup (range 0.1-2.5 THz, dynamic $70 \mathrm{~dB}, 30$ scans second) with external free standing module with a fibre-fed emitter and a detector. $\mathrm{THz}$ beam by means of a set of mirrors was focused on the sample and after reflection was collected and focused on the detector. Incident angle was $7^{\circ}$. It is revealed that reflectance and phase difference can be applied for identification of hexogen in the reflection configuration.

\footnotetext{
* e-mail: npalka@wat.edu.pl
}

\section{Theory}

TDS [1-9] is a commonly used technique in THz range to measure spectral parameters of materials. Generally, it bases on generation and detection of pulses, which last about $1 \mathrm{ps}$ and have broadband spectrum usually in the range $0.1-3.0 \mathrm{THz}$. The detailed description of TDS technique can be found in [4-10].

The main advantage of transmission TDS technique is that one can easily determine the complex refractive index $(N)$ of measured materials

$$
N=n+\mathrm{i} \kappa,
$$

where $n$ is the refractive index and $\kappa$ is the extinction coefficient. Basing on the power spectra of $\mathrm{THz}$ impulses we can calculate $\kappa$. Thanks to phase spectra of impulses, $n$ can be obtained. Figure $1 \mathrm{a}, \mathrm{b}$ presents the refractive index and the extinction coefficient of a hexogen sample determined in TDS system [10].

In real application, only reflection geometry can be applied because $\mathrm{THz}$ radiation cannot transmit through targets thicker than a few millimeter. Interaction of the terahertz wave and surface of the sample depends on its morphology $[10,11]$. Specular reflection takes place when the sample has a smooth surface and diffuse reflection is used for description of samples with a rough surface. Taking into account Eq. (1), for normal incidence the complex reflection coefficient $(\tilde{r})$ of a sample is described by the Fresnel formula as [11]:

$$
\tilde{r}=\frac{N-1}{N+1}=\frac{n^{2}+\kappa^{2}-1}{(n+1)^{2}+\kappa^{2}}+\mathrm{i} \frac{2 \kappa}{(n+1)^{2}+\kappa^{2}},
$$

which has a real and imaginary part. If we assumed $\kappa \ll 1$, the complex reflection coefficient can be divided into the reflectance $(R)$ and the reflectance phase $(\Delta \phi)$ accordingly [10]:

$$
R=|r|^{2} \approx \frac{n-1}{n+1}, \quad \Delta \phi \approx \frac{2 \kappa}{n^{2}-1} .
$$


Therefore, spectral features of the target are observed in both the reflectance and phase changes in the reflection spectrum. Figure 1c,d shows the reflectance of a RDX sample determined theoretically by means of Eq. (3) using $n$ and $\kappa$ from Fig. 1a,b, respectively.
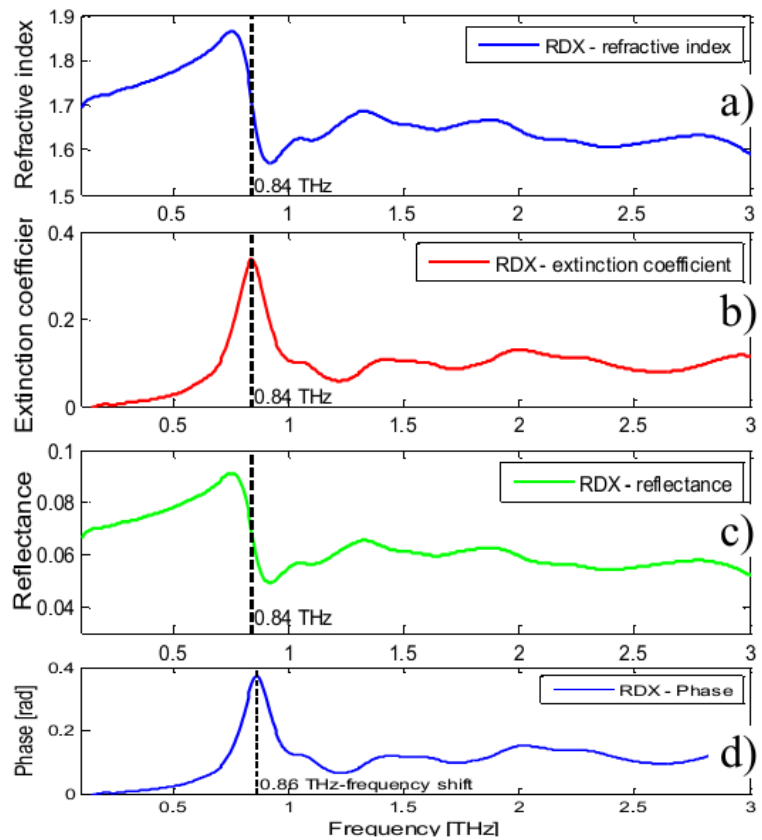

Fig. 1. Refractive index (a), extinction coefficient (b), reflectance (c), and reflectance phase (d) of RDX.

In the reflection spectroscopy, the reflectance phase represents the absorption feature like extinction coefficient in transmission. The reflectance spectrum, however, mimics the refractive index variation.

\section{TDS reflection setup}

Reflection measurements were carried out in an external free standing module from Teraview with a fibre-fed emitter and detector (Fig. 2). THz beam by means of a set of mirrors is focused on the sample and after reflection is collected and focused on the detector. Samples were measured in the compartment purged with dry air (humidity less than 1\%) in the distance $30 \mathrm{~cm}$ between a sample and the mirrors. Incident angle was 7 degrees. The measurement time was a few minutes depending on averaging and length of the scan required for the analysis. The main parameters of the systems are: spectral range 0.3-2.5 THz, signal-to-noise better than 4000:1, dynamic range higher than $3 \mathrm{OD}$ in the range $2 \mathrm{~cm}^{-1}$ to $100 \mathrm{~cm}^{-1}$, spectral resolution $0.06 \mathrm{THz}$ and rapid scan mode with 30 scans/second [7]. Position of the sample and the gold mirror is controlled by means of a micrometer stage.

We firstly measured a reference impulse reflected from the gold mirror and next an impulse reflected from the RDX sample. Afterwards, the impulses were fast Fourier transformed. The reflectance is determined as a ratio of

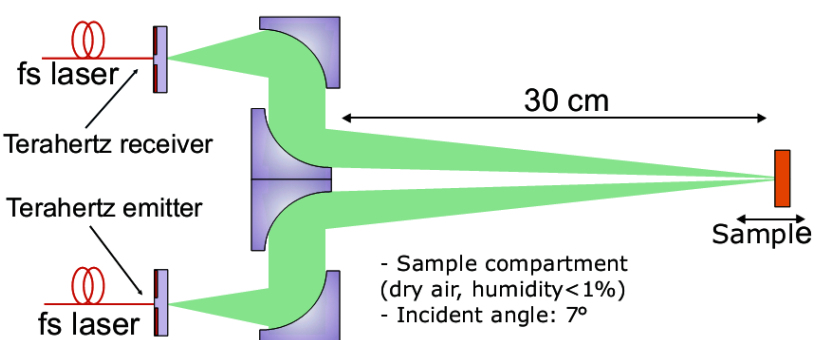

Fig. 2. TDS reflection setup.

power spectra of the sample and the reference impulses, respectively. The reflectance phase can be determined as phase difference between the reference and the sample impulses. In reflection measurements, the reference gold mirror and the sample should be situated exactly in the same position to obtain the best performance. The measured impulses were apodized with the BlackmanHarris 3 term window function to smooth the spectral characteristics.

\section{Samples preparation}

Scanning of pure explosives in transmission mode is limited to samples with thickness less than about $1-2 \mathrm{~mm}$, which is connected with relatively high attenuation especially in peaks regions. In case of thicker samples one should consider the reflection configuration. For these studies, we manufactured pellets which consisted of pure materials with weight $400 \mathrm{mg}$ and $13 \mathrm{~mm}$ diameter. The gold mirror was used as the reference.

We also prepared a mixture of RDX with some plastic binder and plasticizer (similar to C4), to study influence of the sample surface. We applied sandpaper sheets with grit 120, 80 and 40 to extrude random rough surface on the material (Fig. 3a). The relative roughness of sandpaper is indicated by the grit size, which is inversely proportional to the grain size.

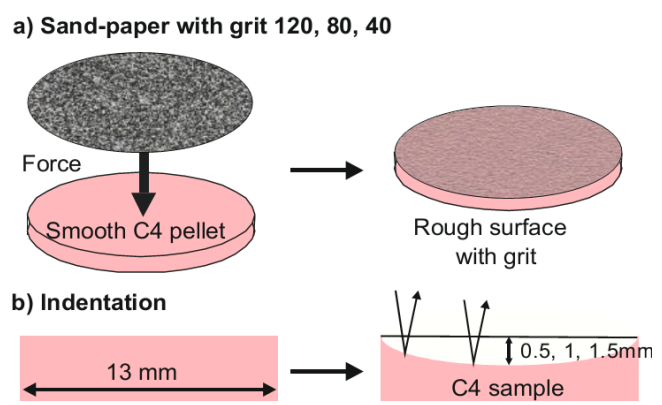

Fig. 3. Schemes of the samples: with rough surface (a) and with indentations (b).

Moreover, to study influence of the real uneven surfaces, in flat and smooth cylinder samples of the plastic RDX with diameter of $13 \mathrm{~mm}$, we created indentations with depth of about 0.5, 1.0, 1.5 mm (Fig. 3b). 


\section{Reflectance measurements}

Terahertz reflection measurements were performed to investigate influence of sample position, surface roughness and curvature on the reflectance and phase.

\subsection{Position of the sample}

According to [12], in reflection measurements, the reference gold mirror and the sample should be situated exactly in the same position to obtain the best performance, which is connected with a so-called misplacement phase error. If the sample and the mirror are not in the same position, $\mathrm{THz}$ radiation travels through different paths and interacts with the different volume of air, which can cause changes in reflectance spectra. To study the influence of the sample position on both reflectance characteristics, we shifted the position of the smooth RDX pellet in the range $-1 \mathrm{~mm}$ up to $3 \mathrm{~mm}$ with respect to a " 0 " position, where the gold mirror was situated. The obtained results - impulses, reflectance and phase spectra are presented in Fig. 4. The theoretical reflectance characteristics from Fig. 1c,d are depicted in Fig. 4b,c for comparison (blue dashed lines).
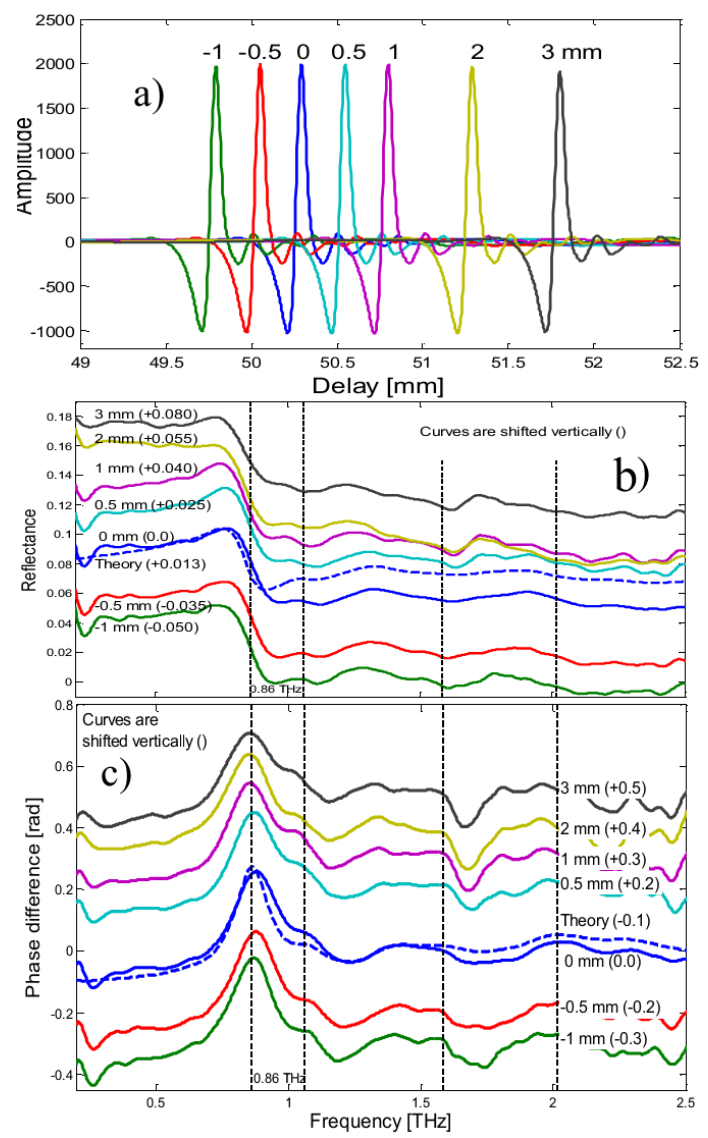

Fig. 4. Impulses (a), reflectances (b), and phase differences (c) of RDX measured for different sample positions. Spectral characteristics were shifted vertically for clarity.
It can be noted that theoretical characteristics (blue dashed lines) are in good agreement with the measured ones (for "0" position) taking into account the position of the peak at $0.86 \mathrm{THz}$ and shape of the curves. We think that the observed discrepancies for reflectance at higher frequencies are connected with higher scattering, because the possible inhomogeneities of the surface are relatively bigger for shorter wavelengths.

It can be also concluded that changes in the position of the sample in the range $-1 \mathrm{~mm}$ up to $3 \mathrm{~mm}$ (detuning) influences the impulses and the reflectance characteristics only in a limited way. The position of the spectral feature at $0.86 \mathrm{THz}$ is clearly seen. Above $1.5 \mathrm{THz}$ some deformation of both reflectance characteristics are observed. However, in the detuning distance $\pm 0.5 \mathrm{~mm}$ both reflectance characteristics are very similar to the " 0 " position in the range up to about $2 \mathrm{THz}$. Nevertheless, further studies with respect to influence of other factors like water vapor are still needed to determine the practical detuning range.

\subsection{Surface roughness}

The measurement results for samples with roughness created by means of sandpapers are presented in Fig. 5 . The samples and the reference mirror were situated in the same position. As expected, the rougher the surface the smaller the reflected signal due to higher scattering on bigger disturbances of the surface. Phase spectrum is more deformed, but we can still easily recognize the characteristic peak of RDX, which is shifted to $0.86 \mathrm{THz}$, which agrees with theoretical considerations (Fig. 1d).

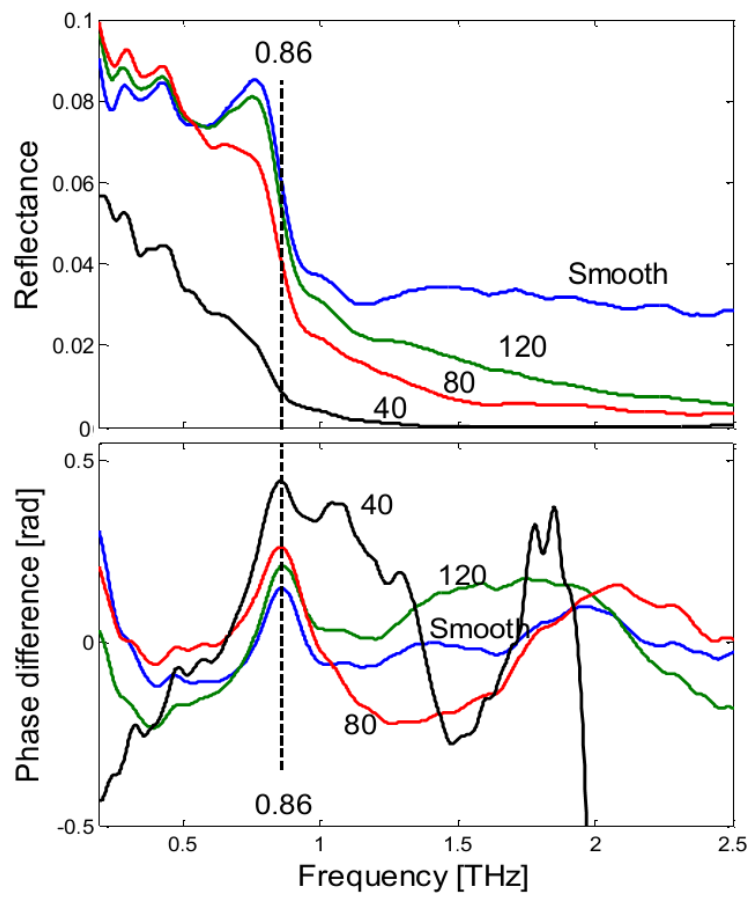

Fig. 5. Reflectance (a) and phase difference (b) characteristics of the plasticized RDX with sandpaper-based rough surfaces. 


\subsection{Surface curvature}

Results of reflection measurements for the samples with curved surfaces (indentation with depth of about $0.5,1,1.5 \mathrm{~mm}$ ) are shown in Fig. 6. The samples and the reference mirror were situated in the same position using the micrometer screw. It is seen that even small surface unevenness caused big decreases in amplitude of the impulses and reflectance spectrum in comparison to the smooth surface. However, the phase spectra changed slightly and only some deformations at higher frequencies were recorded. Figure 6a shows impulses reflected from the curved surfaces. The main short impulses are connected with reflection from a part of indentation, which is perpendicular to the beam. Long blurred impulses before the main impulses originate from the sides of the indentations and their amplitude is smaller. They are rather long in comparison to the main impulses and modify the low frequency range of the phase spectra (Fig. 6c).
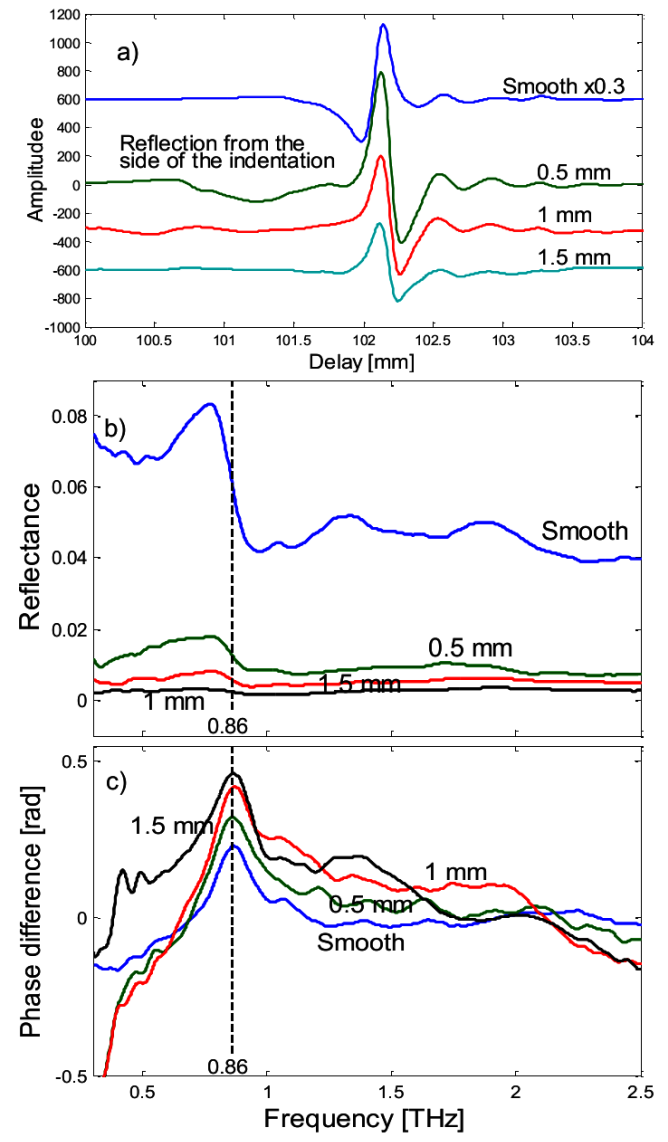

Fig. 6. Amplitudes (a), reflectance (b) and phase difference (c) characteristics of the plasticized RDX with curved surfaces.

\section{Summary}

To sum up, the RDX-based samples with different surfaces and in different positions (detuning) were analyzed using TDS. It can be concluded that both parts of the complex reflectance - amplitude and phase - can be applied for identification of explosives in the reflection configuration. Morover, for samples with modified surfaces, the phase spectrum seems to be more stable the peak of RDX at $0.86 \mathrm{THz}$ is visible and almost unchanged for all measurements, while amplitude features at this frequency can fall down below $1 \%$, which can be hard to measure in real applications. However, further studies with respect to influence of other factors like water vapor are still needed.

These results reveal that $\mathrm{THz}$ reflection measurement can be used for stand-off detection of explosives but strong technological progress is needed in the field of generation and detection of terahertz radiation as well as signal processing to develop commercial systems.

\section{Acknowledgments}

The work was financed by the Polish Ministry of Science and Education under the project O N515 020140.

\section{References}

[1] L. Yun-Shik, Principles of Terahertz Science and Technology, Springer, New York 2008, p. 59.

[2] M.C. Kemp, IEEE Trans. Terahertz Sci. Technol. 1, 282 (2011).

[3] T. Pustelny, J. Ignac-Nowicka, Z. Opilski, Opt. Appl. 34, 563 (2004).

[4] J. Chen, Y. Chen, H. Zhao, G.J. Bastiaans, X.-C. Zhang, Opt. Expr. 15, 12060 (2007).

[5] M.R. Leahy-Hoppa, M.J. Fitch, X. Zheng, L.M. Hayden, R. Osiander, Chem. Phys. Lett. 434, 227 (2007).

[6] N. Palka, Acta Phys. Pol. A 120, 713 (2011).

[7] Teraview Ltd., Presentations and manuals, www . teraview .com (2011).

[8] N. Palka, Acta Phys. Pol. A 118, 1228 (2010).

[9] X.-C. Zhang, J. Xu, Introduction to THz Wave Photonics, Springer, New York 2010, p. 204.

[10] T. Trzcinski, N. Palka, M. Szustakowski, Bull. Pol. Acad. Sci. Tech. 59, 445 (2011).

[11] L.M. Zur, G. Sundberg, S. Schecklman, Z. Zhou, A. Chen, E.I. Thorsos, Proc. SPIE 6949, 694907 (2008).

[12] C. Zhang, H. Zhong, L. Zhang, Proc. SPIE $\mathbf{7 1 5 8}$ 71580Q (2008). 